\title{
Fatores associados aos transtornos depressivos em estudantes universitários da área de saúde: uma revisão integrativa
}

Factors associated with depressive disorders in university students in the health area: an integrative review

Thais Vieira Santos Silva ${ }^{1}$; Luana de Santana Correia ${ }^{1}$; Larissa de Mattos Oliveira ${ }^{1 *}$

${ }^{1}$ Faculdade Maria Milza - FAMAM, Governador Mangabeira - Bahia, Brasil, 44350-000, thais-vss@ @otmail.com; luanasantanacorreia2015@gmail.com; ${ }^{*}$ lare_oliveira@yahoo.com.br; https://orcid.org/0000-0002-6387-8035

\section{Resumo}

A depressão é um transtorno psíquico que se apresenta como uma das principais causas de incapacitação em mais de 300 milhões de pessoas no mundo. Entre essa população, os estudantes da área da saúde têm apresentado cada vez mais transtornos depressivos, o que pode acarretar em prejuízos no desempenho acadêmico e nos relacionamentos interpessoais. Por esse motivo, a saúde mental dos estudantes universitários tem sido foco da atenção de especialistas, visto que estudantes da área de saúde nem sempre recebem informações suficientes sobre saúde mental e se expõem frequentemente a situações de estresse, levando-os ao adoecimento psíquico e dificuldades no atendimento aos pacientes. O objetivo desse estudo foi investigar, por meio de uma revisão integrativa, os fatores descritos na literatura associados aos transtornos depressivos em estudantes da área de saúde. Os estudos analisados mostram uma associação da má qualidade do sono, falta de lazer e atividade física, alimentação inadequada, influência familiar, uso de substâncias ilícitas, dificuldades financeiras, o fato de ser mulher e aspectos acadêmicos, como fatores predisponentes para a depressão entre essa população.

Palavras chave: depressão, estudantes universitários, estudantes de ciências da saúde. 


\begin{abstract}
Depression is a mental disorder that presents itself as one of the main causes of disability in more than 300 million people worldwide. Among this population, students in the health field have increasingly presented depressive disorders, which can result in losses in academic performance and interpersonal relationships. For this reason, the mental health of university students has been the focus of the attention of specialists, since students in the health field do not always receive enough information about mental health and are frequently exposed to stressful situations, leading them to psychic illness and difficulties in patient care. The aim of this study was to investigate, through an integrative review, the factors described in the literature associated with depressive disorders in health students. The analyzed studies show an association between poor sleep quality, lack of leisure and physical activity, inadequate diet, family influence, use of illegal substances, financial difficulties, the fact of being a woman and academic aspects, as predisposing factors for depression among this population..
\end{abstract}

Keywords: depression, university students, health science students.

\title{
1. Introdução
}

A depressão é um transtorno psíquico que afeta as condições afetivas e sociais de um indivíduo e apresenta-se como uma das principais causas de incapacitação no mundo, constituindo um grave problema de saúde pública por sua alta prevalência, limitando o funcionamento físico, pessoal e social do indivíduo (Gonçalves et al., 2017). A doença acomete mais de 300 milhões de pessoas em todo o mundo e é a causa de 800 mil mortes por suicídio a cada ano, sendo a segunda principal causa de morte entre jovens de 15 a 29 anos (OPAS/OMS BRASIL, 2018).

A depressão ou 'transtorno depressivo maior' (TDM) é caracterizada pelo predomínio do humor deprimido ou perda de interesse ou prazer em exercer diversas atividades. Além disso, pode apresentar outros sintomas, como: sentimento de culpa ou desvalia; alterações no sono, peso, apetite e mudanças na atividade psicomotoras; dificuldade para concentrar-se, tomar decisões ou pensar e apresentar pensamentos frequentes sobre morte ou suicídio (Uher et al., 2014; Ménard et al., 2016). Existe, ainda, a correlação entre a depressão e outras morbidades como diabetes, obesidade, problemas oncológicos e cardiopatias, sendo ainda associada a outras situações como depressão na maternidade, problemas no desenvolvimento infantil, complicações no ambiente familiar e piora no rendimento escolar (Gonçalves et al., 2017). 
Para o tratamento da depressão, deve ser considerado todo o suporte terapêutico, desde a prática de atividades físicas, terapia cognitivo-comportamental e psicoterapia interpessoal, até o uso de medicamentos antidepressivos, visando diminuir os sintomas, recuperar a capacidade funcional e melhorar a qualidade de vida (Silva et al., 2012; OPAS BRASIL, 2018).

Adicionalmente, pessoas do sexo feminino, pessoas divorciadas ou separadas, viúvas ou que moram sozinhas, apresentam idade entre 20 e 40 anos, possuem baixa renda e baixa escolaridade, não têm suporte social, residem em zona urbana e estão expostas a estresse, têm maior tendência aos transtornos depressivos (Rombaldi et al., 2010).

Particularmente, a população jovem estudantil apresenta-se com transtornos depressivos com mais frequência do que na população geral, acarretando em prejuízos no desempenho acadêmico (Cybulski \& Mansani, 2017). Durante a graduação, cerca de 15\% a 25\% dos estudantes apresentam quadros de transtornos psíquicos (Segall, 1966; Giglio, 1975; Eric et al., 1988), devido aos fatores de estresse que envolvem alto nível de cobrança pessoal, familiar e institucional, sobrecarga de conteúdo, afastamento dos pais, expectativas sociais e pouco tempo para descanso (Fiorotti et al., 2010).

Neste contexto, esse estudo tem como objetivo investigar, por meio de uma revisão integrativa, os fatores descritos na literatura associados aos transtornos depressivos em estudantes da área de saúde.

\section{Material e Métodos}

O presente trabalho trata-se de uma revisão integrativa. Com o objetivo de investigar a associação entre a depressão e possíveis fatores relacionados ao ensino superior em estudantes da área da saúde. A pergunta da pesquisa foi construída a partir do acrônimo PICO Participantes, Intervenção, Comparador, Outcome/Desfecho (Santos et al., 2007).

P (Participantes): Pesquisas sobre depressão em estudantes da área de saúde do ensino superior.

I (Intervenção): Diagnóstico clínico ou prevalência de depressão em estudantes da área de saúde do ensino superior. 
C (Comparador): Sem comparador.

O (Outcome/Desfecho): Foram estabelecidas como desfechos quaisquer medidas de associação para a prevalência de depressão em estudantes da área de saúde do ensino superior.

A partir da pergunta "Quais são os fatores predisponentes para os transtornos depressivos em estudantes universitários da área de saúde?", foram realizadas pesquisas individualizadas em bases de dados (MEDLINE - PubMed) através da combinação de palavras-chave: depression e university students, no período compreendido entre 2015 a 2020 e sem restrição de idioma.

Para a realização desta revisão, foram incluídos os estudos quantitativos que associaram a ocorrência de transtornos depressivos em estudantes universitários da área de saúde. Por outro lado, foram excluídos estudos nos quais não foi possível extrair dados relevantes como características da população descrita, além de estudos nos quais o texto completo não estava disponível.

\section{Resultados}

Foram selecionados 55 artigos compatíveis com o tema do estudo, os quais estavam disponíveis na base de dados MEDLINE (PubMed) e 1 artigo adicionado manualmente. Destes artigos, 44 foram utilizados e 12 foram excluídos após a leitura do texto completo. Dos artigos remanescentes, 40 trabalhos foram estudos transversais, 3 revisões sistemáticas e 1 estudo longitudinal (Figura 1). Nesses estudos, pode-se observar a alta prevalência de depressão entre os estudantes universitários e diversos fatores que estão associados à vida acadêmica. Os dados obtidos com o levantamento dos estudos são mostrados no Quadro 1. 


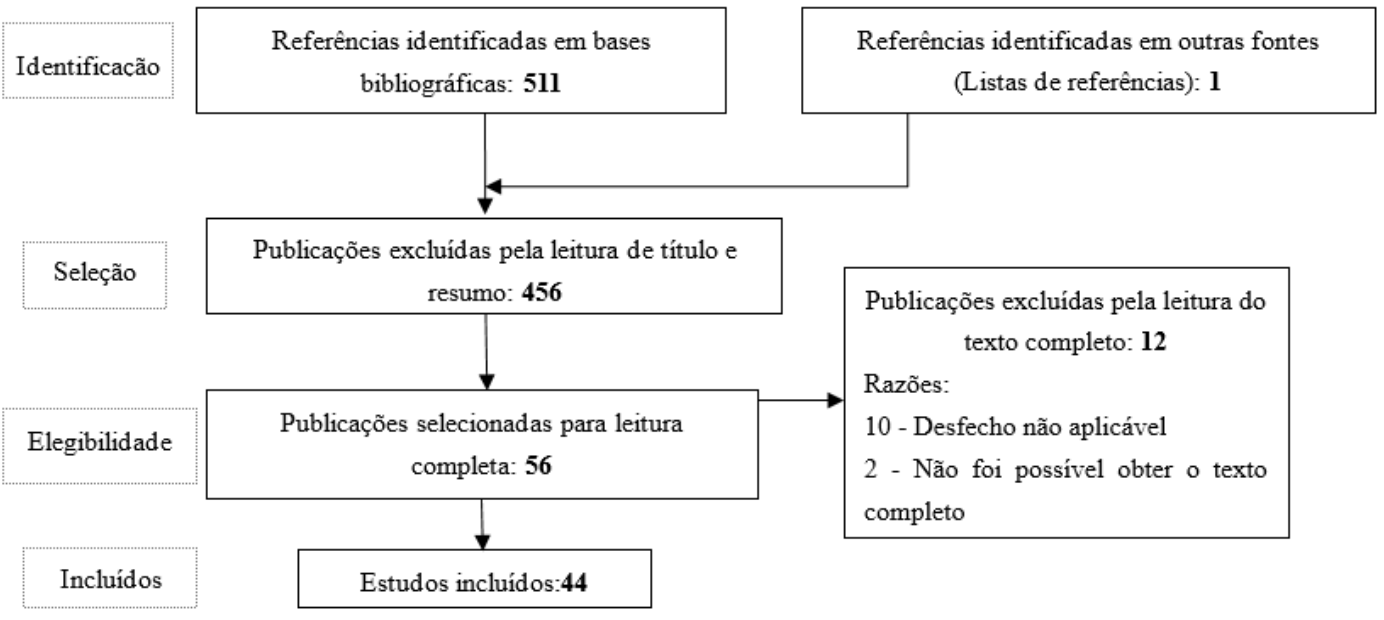

Figura 1. Fluxograma de seleção dos estudos. 
Quadro 1 - Caracterização dos estudos incluídos.

\begin{tabular}{|c|c|c|}
\hline $1^{\circ}$ Autor (ano) & População & Desfechos avaliados \\
\hline $\begin{array}{l}\text { Arbués et al. } \\
\text { (2019) }\end{array}$ & $\begin{array}{l}1.055 \\
\text { estudantes } \\
\text { universitários }\end{array}$ & $\begin{array}{l}\text { A alimentação não saudável foi significativamente associada à prevalência de ansiedade, depressão e estresse; oconsumo } \\
\text { excessivo de doces e o déficit de consumo de laticínios foram associados a maior prevalência de distúrbios psicológicos e do } \\
\text { sono. }\end{array}$ \\
\hline Badrasawi. (2019) & $\begin{array}{l}154 \text { estudantes } \\
\text { universitárias }\end{array}$ & $\begin{array}{l}\text { Os sintomas da compulsão alimentar estão significativamente relacionados ao estresse; há associação significativa entre } \\
\text { compulsão alimentar e depressão. }\end{array}$ \\
\hline Chan. (2020) & $\begin{array}{l}655 \text { estudantes } \\
\text { universitários }\end{array}$ & $\begin{array}{l}\text { Estudantes do sexo masculino, estudantes de medicina e aqueles que estudam em programas de cinco anos apresentaram níveis } \\
\text { significativamente mais altos de depressão, ansiedade e estresse; estudantes universitários com níveis mais altos de crenças } \\
\text { irracionais eram mais propensos a ter depressão, ansiedade e estresse. }\end{array}$ \\
\hline $\begin{array}{l}\text { Cheung et al. } \\
\text { (2016) }\end{array}$ & $\begin{array}{l}661 \text { estudantes } \\
\text { universitários }\end{array}$ & $\begin{array}{l}\text { A depressão foi associada com o ano de estudo; especialidade clínica; dificuldades financeiras; crises de relacionamento; fatores } \\
\text { de estilo de vida, incluindo falta de dieta equilibrada, exercícios físicos, entretenimento, hobbies; problemas de sono; percepção } \\
\text { da saúde física e mental. }\end{array}$ \\
\hline Cuttilan. (2016) & N.A & $\begin{array}{l}\text { Estudos têm demonstrado que o conhecimento dos estudantes de medicina em questões de saúde mental, bem como por meio de } \\
\text { intervenção psicológica, resulta nessas taxas de prevalência mais baixas em comparação aos estudantes de outros cursos de } \\
\text { graduação. }\end{array}$ \\
\hline Dagnew. (2020) & $\begin{array}{l}383 \text { estudantes } \\
\text { universitários }\end{array}$ & $\begin{array}{l}\text { A chance de ter depressão foi maior entre os estudantes oriundos de familias rurais, que sofreram ranger de dentes, com } \\
\text { distúrbios do sono, que relataram sonolência diurna, estresse e aqueles que estudam ciências da saúde. }\end{array}$ \\
\hline Delara. (2015) & $\begin{array}{l}171 \text { estudantes } \\
\text { universitários }\end{array}$ & $\begin{array}{l}\text { Estudantes de ciências da saúde são sujeitos ao estresse de fontes possivelmente relacionadas ao seu treinamento educacional, } \\
\text { resultando em problemas significativos de saúde mental. }\end{array}$ \\
\hline Desouky (2019) & $\begin{array}{c}1.340 \\
\text { estudantes } \\
\text { universitárias } \\
\text { do sexo } \\
\text { feminino }\end{array}$ & $\begin{array}{l}\text { A prevalência de depressão foi significativamente maior entre migrantes e estudantes que sofreram frequências mais altas de dor } \\
\text { de cabeça; a prevalência de depressão foi significativamente maior entre os estudantes com enxaqueca. }\end{array}$ \\
\hline
\end{tabular}


Continuação... Quadro 1 - Caracterização dos estudos incluídos.

\begin{tabular}{|c|c|c|}
\hline $1^{\circ}$ Autor (ano) & População & Desfechos avaliados \\
\hline Dinis. (2018) & N.A & $\begin{array}{l}\text { Fatores genéticos, ano de estudo, uso da tecnologia, cronótipo, quantidade do sono são fatores que influenciam a qualidade do } \\
\text { sono e a depressão. }\end{array}$ \\
\hline $\begin{array}{l}\text { Džubur et al. } \\
\text { (2018) }\end{array}$ & $\begin{array}{l}412 \text { estudantes } \\
\text { universitários }\end{array}$ & $\begin{array}{l}\text { Os fatores associados ao aumento de depressão dos estudantes de medicina foram: acomodações em dormitórios, não realizam } \\
\text { atividade física e abuso de substâncias. }\end{array}$ \\
\hline $\begin{array}{l}\text { Fernandes et al. } \\
\text { (2018) }\end{array}$ & $\begin{array}{l}205 \text { estudantes } \\
\text { universitários }\end{array}$ & Identificou-se associação entre o nível de sintomas depressivos, trabalho, sexo e lazer. \\
\hline Flesch et al. (2020) & $\begin{array}{l}1.827 \\
\text { estudantes } \\
\text { universitários }\end{array}$ & $\begin{array}{l}\text { A depressão era mais frequente entre as mulheres, com idades entre } 21 \text { e } 23 \text { anos, com histórico familiar de depressão, } \\
\text { homossexuais e bissexuais, que moravam com amigos e colegas; estudantes da área de ciências sociais e humanas aplicadas, bem } \\
\text { como linguística, língua e literatura e artes. O pior desempenho acadêmico, o abuso de álcool e o uso de drogas ilícitas também } \\
\text { foram associados à depressão. }\end{array}$ \\
\hline $\begin{array}{l}\text { Grasdalsmoen et } \\
\text { al. (2020) }\end{array}$ & $\begin{array}{l}50.054 \\
\text { estudantes } \\
\text { universitários }\end{array}$ & $\begin{array}{l}\text { A duração e a intensidade do exercício físico foram significativamente associadas a problemas de saúde mental; quanto menos } \\
\text { frequente o exercício físico dos alunos, maior a prevalência de depressão. }\end{array}$ \\
\hline Gul et al. (2020) & $\begin{array}{l}1.159 \\
\text { estudantes } \\
\text { universitários }\end{array}$ & $\begin{array}{l}\text { A depressão no sexo feminino foi significativamente maior que no sexo masculino; a depressão teve uma associação significativa } \\
\text { com as notas acadêmicas. }\end{array}$ \\
\hline Hakami. (2018) & $\begin{array}{l}450 \text { estudantes } \\
\text { universitários }\end{array}$ & As mulheres relataram mais sintomas de somatização, depressão e ansiedade do que os homens \\
\hline Hambisa. (2020) & $\begin{array}{l}1.022 \\
\text { estudantes } \\
\text { universitários }\end{array}$ & $\begin{array}{l}\text { Consumo de álcool, tabagismo, uso de substâncias ilícitas; o primeiro ano de universidade pode ser um período de adaptação } \\
\text { particularmente vulnerável para os alunos; os que se divorciaram / viúvos têm associação significativa com sintomas depressivos. }\end{array}$ \\
\hline Kilinç et al. (2020) & $\begin{array}{l}1.004 \\
\text { estudantes } \\
\text { universitários }\end{array}$ & Houve uma relação positiva entre os níveis de solidão e depressão entre estudantes universitários. \\
\hline
\end{tabular}


Continuação... Quadro 1 - Caracterização dos estudos incluídos.

\begin{tabular}{|c|c|c|}
\hline $1^{\circ}$ Autor (ano) & População & Desfechos avaliados \\
\hline Knipe et al. (2018) & $\begin{array}{l}1.139 \\
\text { estudantes } \\
\text { universitários }\end{array}$ & $\begin{array}{l}\text { Apenas } 21 \% \text { dos estudantes com sintomas de depressão grave procuraram ajuda profissional; a principal razão para não procurar } \\
\text { ajuda foi o medo de documentação nos registros acadêmicos. }\end{array}$ \\
\hline Leão et al. (2018) & $\begin{array}{l}476 \text { estudantes } \\
\text { universitários }\end{array}$ & $\begin{array}{l}\text { Estudantes menos satisfeitos com o curso apresentaram chance quase quatro vezes maior de terem depressão. Destacaram-se } \\
\text { ainda fatores de risco como relacionamento familiar insatisfatório, sono insuficiente, relacionamento insatisfatório com colegas e } \\
\text { falta de atividade física. }\end{array}$ \\
\hline Lee (2019) & $\begin{array}{l}244 \text { estudantes } \\
\text { universitários }\end{array}$ & $\begin{array}{l}\text { O comportamento sedentário de estudantes universitários mostrou-se significativamente relacionado aos seus problemas } \\
\text { psicológicos. }\end{array}$ \\
\hline Lei et al. (2016) & N.A & $\begin{array}{l}\text { Maior prevalência de depressão entre estudantes de medicina. Estudantes expostos a situações negativas como falha no amor, } \\
\text { separação dos pais e déficits financeiros, eram fonte direta de estímulo que induzia a depressão. }\end{array}$ \\
\hline Li et al. (2015) & $\begin{array}{l}1.843 \\
\text { estudantes } \\
\text { universitários }\end{array}$ & $\begin{array}{l}\text { Estrutura familiar, experiência negativa na infância e adolescência e fatores socioambientais na família de origem do aluno } \\
\text { correlacionaram-se significativamente com a prevalência de TDM ao longo da vida. }\end{array}$ \\
\hline López (2017) & $\begin{array}{l}235 \text { estudantes } \\
\text { universitários }\end{array}$ & $\begin{array}{l}\text { Os dados encontrados evidenciam que as dimensões da organização da personalidade teriam uma relação significativa com a } \\
\text { presença de depressão, ansiedade e estresse. }\end{array}$ \\
\hline Lun et al. (2018) & $\begin{array}{l}1.119 \\
\text { estudantes } \\
\text { universitários }\end{array}$ & $\begin{array}{l}\text { Os alunos com exercícios regulares, maior autoconfiança, melhor satisfação com o desempenho acadêmico e mais otimismo em } \\
\text { relação ao futuro experimentaram menos sintomas depressivos. }\end{array}$ \\
\hline $\begin{array}{l}\text { Mboya et al. } \\
\text { (2020) }\end{array}$ & $\begin{array}{l}402 \text { estudantes } \\
\text { universitários }\end{array}$ & $\begin{array}{l}\text { Área de residência, histórico familiar de doença mental, disponibilidade de apoio social e diminuição da nota do que o previsto } \\
\text { foram os fatores que se mostraram associados significativamente ao sofrimento mental. }\end{array}$ \\
\hline Milic et al. (2020) & $\begin{array}{l}2.138 \\
\text { estudantes } \\
\text { universitários }\end{array}$ & $\begin{array}{l}\text { A associação do tabagismo com a diminuição da qualidade de vida relacionada à saúde entre estudantes universitários foi } \\
\text { mediada por níveis mais altos de sintomas depressivos. }\end{array}$ \\
\hline
\end{tabular}


Continuação... Quadro 1 - Caracterização dos estudos incluídos.

\begin{tabular}{|c|c|c|}
\hline $1^{\circ}$ Autor (ano) & População & Desfechos avaliados \\
\hline Mitchell. (2018) & $\begin{array}{l}121 \text { estudantes } \\
\text { universitários }\end{array}$ & $\begin{array}{l}\text { As principais conclusões mostram altos níveis de sofrimento psicológico nos estudantes de enfermagem, acima dos níveis } \\
\text { observados na população em geral. }\end{array}$ \\
\hline Nahas et al. (2019) & $\begin{array}{l}365 \text { estudantes } \\
\text { universitários }\end{array}$ & $\begin{array}{l}\text { As mulheres apresentaram taxas mais altas de nível grave de depressão; a maioria dos estudantes das áreas urbanas tinha maior } \\
\text { probabilidade de relatar sintomas depressivos de várias gravidades; os estudantes universitários de ciências da saúde estão em } \\
\text { maior risco de depressão. }\end{array}$ \\
\hline Ngin et al. (2018) & $\begin{array}{l}1.359 \text { estudantes } \\
\text { universitários }\end{array}$ & $\begin{array}{l}\text { Os sintomas depressivos permaneceram significativamente associados ao baixo desempenho acadêmico, maior consumo de } \\
\text { alimentos não saudáveis, uma autopercepção negativa sobre a forma corporal, estado geral de saúde e atividade física limitada, } \\
\text { experiências adversas na infância, incluindo violência física, abuso psicológico e falta de cuidados gerais e médicos. }\end{array}$ \\
\hline $\begin{array}{l}\text { Othman et al. } \\
\text { (2019) }\end{array}$ & $\begin{array}{l}148 \text { estudantes } \\
\text { universitários }\end{array}$ & $\begin{array}{l}\text { Vários determinantes pareciam ter um alto impacto negativo percebido no bem-estar dos alunos, como encontrar um emprego } \\
\text { desejado, atender às expectativas da família, conflito familiar, sentindo-se julgado, pagando propinas, exclusão social e } \\
\text { obtenção da nota desejada. }\end{array}$ \\
\hline Oyekcin (2017) & $\begin{array}{l}430 \text { estudantes } \\
\text { hiversitários }\end{array}$ & Verificou-se que a gravidade dos sintomas mentais aumentava com a diminuição da percepção da saúde física e mental. \\
\hline Oztasan. (2016) & $\begin{array}{l}429 \text { estudantes } \\
\text { universitários }\end{array}$ & $\begin{array}{l}\text { Os problemas mentais dos alunos aumentam e suas qualidades de vida diminuem; a depressão não apenas diminui a atenção, } \\
\text { concentração, memória, motivação, habilidades de tomada de decisão, mas também é um fator de risco para tentativa de } \\
\text { suicídio. }\end{array}$ \\
\hline Piumatti (2018) & $\begin{array}{l}228 \text { estudantes } \\
\text { de graduação }\end{array}$ & $\begin{array}{l}\text { Indivíduos altamente motivados positivamente são menos propensos a apresentar sintomas de sofrimento psicológico; variáveis } \\
\text { como fatores de vida e estilo de vida, incluindo tabagismo, consumo de álcool, uso de substâncias, satisfação com a escolha } \\
\text { acadêmica e o estresse financeiro, contribuem para a probabilidade de depressão. }\end{array}$ \\
\hline Rehmani (2018) & $\begin{array}{l}283 \text { estudantes } \\
\text { universitários }\end{array}$ & $\begin{array}{l}\text { Os fatores identificados como estressores incluíram pressão para passar no exame, atender às expectativas da família de bom } \\
\text { desempenho acadêmico e falta de casa. }\end{array}$ \\
\hline Rossi et al. (2019) & $\begin{array}{l}580 \text { estudantes } \\
\text { universitários }\end{array}$ & $\begin{array}{l}\text { As mulheres apresentaram escores mais altos nas relações positivas e propósito nas dimensões da vida; a alta prevalência de } \\
\text { sintomas depressivos nos sujeitos deste estudo está relacionada a condições típicas da vida universitária. }\end{array}$ \\
\hline
\end{tabular}


Continuação... Quadro 1 - Caracterização dos estudos incluídos.

\begin{tabular}{|c|c|c|}
\hline $1^{\circ}$ Autor (ano) & População & Desfechos avaliados \\
\hline $\begin{array}{l}\text { Ruisoto et al. } \\
\text { (2016) }\end{array}$ & $\begin{array}{l}3.232 \\
\text { estudantes } \\
\text { universitários }\end{array}$ & $\begin{array}{l}\text { Nos homens, o perfil de consumo problemático de álcool foi definido por escores mais altos de ansiedade e depressão, } \\
\text { principalmente se eles apresentaram níveis mais altos de estresse psicológico e menor envolvimento na vida. Nas mulheres, o } \\
\text { consumo problemático mostrou uma tendência à inflexibilidade psicológica, principalmente naquelas com menor envolvimento } \\
\text { na vida. }\end{array}$ \\
\hline Santangelo (2018) & $\begin{array}{l}1.102 \\
\text { estudantes } \\
\text { universitários }\end{array}$ & $\begin{array}{l}\text { Os dados mostram que o risco de ansiedade, depressão e alcoolismo tem uma distribuição semelhante em todos os cursos de } \\
\text { graduação; as mulheres são mais vulneráveis à ansiedade e à depressão; o consumo arriscado de álcool está associado } \\
\text { significativamente a um risco aumentado de sintomas depressivos moderados, graves ou muito graves e vice-versa. }\end{array}$ \\
\hline $\begin{array}{l}\text { Schlarb et al. } \\
\text { (2017) }\end{array}$ & $\begin{array}{l}2.831 \\
\text { estudantes } \\
\text { universitários }\end{array}$ & $\begin{array}{l}\text { Os estudantes universitários apresentaram sintomatologia depressiva ou de estresse moderada ou grave e relataram um nível } \\
\text { alarmante de distúrbios do sono. }\end{array}$ \\
\hline Seo et al. (2018) & $\begin{array}{l}187 \text { estudantes } \\
\text { universitários }\end{array}$ & Foi encontrada uma forte associação significativamente positiva entre sintomas depressivos e estresse percebido. \\
\hline $\begin{array}{l}\text { Sibnath et al. } \\
\text { (2016) }\end{array}$ & $\begin{array}{l}717 \text { estudantes } \\
\text { universitários }\end{array}$ & $\begin{array}{l}\text { O ambiente familiar e relacionamento com os pais tinha um impacto positivo nos níveis de depressão; A depressão foi impactada } \\
\text { pelo semestre (o semestre anterior foi mais desafiador do que os semestres posteriores), disciplina, status e desempenho } \\
\text { acadêmico; Estudantes de ciências humanas e sociais sofriam mais depressão em comparação com estudantes de ciências e de } \\
\text { gestão. }\end{array}$ \\
\hline $\begin{array}{l}\text { Supartini et al. } \\
\text { (2016) }\end{array}$ & $\begin{array}{l}1.992 \\
\text { estudantes } \\
\text { universitários }\end{array}$ & $\begin{array}{l}\text { Os sintomas depressivos estavam intimamente associados com a hora de dormir mais tarde, latência prolongada no início do sono } \\
\text { e má qualidade do sono; a ideação suicida foi significativamente associada apenas à baixa qualidade do sono, independentemente } \\
\text { dos sintomas depressivos. }\end{array}$ \\
\hline Tayefi et al. (2020) & $\begin{array}{l}560 \text { estudantes } \\
\text { universitários }\end{array}$ & $\begin{array}{l}\text { Etnia, disciplina, local de nascimento e escolaridade materna foram os principais determinantes da saúde mental entre estudantes } \\
\text { de ciências da saúde. }\end{array}$ \\
\hline Tran et al. (2017) & $\begin{array}{l}4.184 \\
\text { estudantes de } \\
\text { graduação }\end{array}$ & $\begin{array}{l}\text { Os estudantes insatisfeitos com suas condições de vida e aqueles com mau comportamento alimentar estavam em risco de } \\
\text { depressão; os que apresentavam dificuldades financeiras e de aprendizagem foram associados a sintomas psiquiátricos; ser } \\
\text { mulher e morar sozinha estava associado à ansiedade. }\end{array}$ \\
\hline Tuyen. (2019) & $\begin{array}{l}405 \text { estudantes } \\
\text { universitários }\end{array}$ & $\begin{array}{l}\text { Economia familiar, consumo de álcool, ano de estudo e preocupação com futuras carreiras após a graduação, foram variáveis } \\
\text { independentes significativas na prevalência de sintomas depressivos entre os estudantes. }\end{array}$ \\
\hline
\end{tabular}




\section{Discussão}

Durante o período universitário a saúde mental dos estudantes pode estar fragilizada diante dos muitos obstáculos e fatores estressantes ao longo dessa fase (Santagelo, 2018). Com isso os alunos desenvolvem hábitos prejudiciais à saúde mental e existem também fatores externos que contribuem para o aparecimento de sintomas depressivos.

Dentre os hábitos prejudiciais está o consumo de álcool. Dos estudos analisados, em seis deles (Santangelo, 2018; Flesch et al., 2020; Piumatti, 2018; Hambisa, 2020; Tuyen, 2019; Ruisoto et al., 2016), esse hábito está associado significativamente a um risco aumentado de sintomas depressivos que pode ser atribuído à mudança no estilo de vida dos estudantes. Além do consumo de álcool, o tabagismo está associado com a diminuição da qualidade de vida relacionada à saúde dos estudantes universitários, resultando em níveis mais altos de sintomas depressivos (Milic et al., 2020). Três estudos (Piumatti, 2018; Hambisa, 2020; Milic et al., 2020) mostraram essa associação.

Os alunos que fizeram o uso de substâncias ilícitas mostraram predisponência para a depressão (Piumatti, 2018). No estudo de Flesh et al. (2020), $23 \%$ dos participantes da pesquisa usaram substâncias ilícitas. No estudo de Hambisa (2020), aqueles que usaram substâncias ilícitas tiveram 2,20 vezes mais chances de desenvolver sintomas depressivos do que aqueles que não usaram. Nos resultados de Tran et al. (2017), $10 \%$ dos estudantes fumavam maconha. Džubur et al. (2018) e Milic et al. (2020) relataram que alguns indivíduos abusam de substâncias pensando, erroneamente, que tal ato pode aliviar e facilitar lidar com o estresse contínuo durante o período acadêmico.

Quanto ao sexo, diante dos dados analisados, as mulheres apresentam maior prevalência de depressão, sendo evidente que o sexo feminino está mais propenso ao desenvolvimento de sintomas depressivos (Santangelo, 2018; Hakami, 2018; Fernandes et al., 2018; Rossi et al., 2019; Lee, 2019; Nahas et al., 2019; Flesch et al., 2020). De acordo com Hakami, (2018) e Nahas et al. (2019), as diferenças de gênero na prevalência de problemas de saúde mental podem ser explicadas por fatores psicossociais e biológicos, devido, por exemplo, à maior sensibilidade às relações interpessoais, bem como às alterações hormonais.

Outro fator observado foi que a alimentação não saudável dos estudantes foi associada à 
prevalência de depressão (Cheung et al., 2016; Tran et al., 2017; Ngin, 2018; Arbués et al., 2019). No estudo realizado por Badrasawi (2019), $50 \%$ das estudantes apresentaram sintomas de compulsão alimentar. Estes sintomas estão relacionados com o estresse e foram associados com a depressão. Segundo Arbués et al. (2019), a prevalência de distúrbios do bem-estar psicológico e do sono está associada à falta de alimentação saudável e falta de adesão às recomendações para ingestão de legumes, verduras, consumo de laticínios deficiente e o consumo excessivo de doces.

No quesito sono, devido às mudanças de rotina na vida dos estudantes universitários podem-se desenvolver problemas de sono e resultar em maiores estresses emocionais, o que acarretaria em níveis mais elevados de sintomas depressivos (Schlarb et al., 2017). De acordo com os estudos analisados (Cheung et al., 2016; Schlarb et al., 2017; Dinis, 2018; Dagnew, 2020), a chance de ter depressão foi maior em estudantes com distúrbios do sono. Os resultados do estudo de Schlarb et al. (2017) descreveram que 42,8\% dos estudantes tiveram a qualidade do sono prejudicada, 25,5\% relataram depressão elevada e $45 \%$ indicaram níveis elevados de estresse. Esse estudo demonstrou que os estudantes que apresentaram sintomatologia depressiva relataram altos níveis de distúrbios do sono.

A quantidade de sono insuficiente também se mostrou como fator predisponente para depressão (Leão et al., 2018). Para Supartini et al. (2016), os sintomas depressivos estavam intimamente associados com a hora de dormir (mais tarde), latência prolongada no início do sono e má qualidade do sono. A ideação suicida foi associada apenas à baixa qualidade do sono.

Um outro fator associado aos sintomas depressivos é a falta de exercício físico (Cheung et al., 2016; Ngin et al., 2018; Džubur et al., 2018). Segundo Lee (2019), o comportamento sedentário durante os dias da semana e fins de semana teve um efeito significativo na depressão e os participantes obesos apresentaram maior prevalência de depressão. No estudo de Ngin et al. (2018), fatores como a autopercepção negativa sobre a forma corporal também se apresenta como associação aos sintomas depressivos. Para Grasdalsmoen et al. (2020), quanto menos frequente o exercício físico dos alunos, maior a prevalência de depressão. Sibnath et al. (2016) e Lun et al. (2018) afirmam que fazer exercícios regulares contribui positivamente para a manutenção da saúde mental. 
A falta de lazer também foi apontada como fator associado à depressão (Cheung et al., 2016; Fernandes et al., 2018). Esses fatores podem estar relacionados à falta de tempo livre devido à grande demanda de atividades curriculares.

Estrutura familiar, experiência negativa na infância e adolescência e fatores socioambientais na família de origem do aluno também se correlacionaram significativamente com a prevalência de depressão ao longo da vida (Li et al., 2015), bem como a economia familiar (Tuyen, 2019). Estudos demonstram que o conflito familiar é um dos fatores associados à depressão nos estudantes (Othman et al., 2019; Cheung et al., 2016; Leão et al., 2018). Os estudos mostram que o ambiente familiar e relacionamento com os pais é um fator que contribui para o aumento dos níveis de depressão (Sibnath et al., 2016).

Histórico familiar de doença mental também se mostrou associado à prevalência de sintomas depressivos (Mboya et al., 2020). No estudo de Flesch et al. (2020), 56\% dos alunos relataram ter história familiar de depressão e nos resultados de Rehmani (2018), 14\% dos alunos têm um histórico familiar positivo de depressão. Além disso, a família, ao depositar suas expectativas, tem alto impacto negativo na saúde mental do estudante (Rehmani, 2018; Othman et al., 2019; Chan, 2020).

A qualidade de vida inclui aspectos como atender às necessidades básicas e expectativas sociais de um indivíduo e se beneficiar das oportunidades oferecidas pela sociedade em que ele vive (Oztasan et al., 2016). De acordo com os resultados de Kilinç et al. (2019), a situação econômica foi um indicador importante para depressão. Dessa forma, os estudantes que apresentaram dificuldades financeiras tiveram maior probabilidade de ter depressão (Lei et al., 2016; Cheung et al., 2016; Tran et al., 2017; Piumatti 2018; Gul et al., 2020).

Os aspectos acadêmicos também influenciam a ocorrência de depressão entre os estudantes universitários e os sintomas depressivos permaneceram significativamente associados ao baixo desempenho acadêmico (Sibnath et al., 2016; Ngin et al., 2018; Flesch et al., 2020), à obtenção de notas indesejadas (Othman et al., 2019; Mboya et al., 2020; Gul et al., 2020), menor satisfação com a escolha acadêmica (Piumatti, 2018; Leão et al., 2018), além de não estar no curso escolhido como primeira opção (Leão et al., 2018) e à preocupação em encontrar um emprego (Othman et al., 2019). A maior prevalência de depressão pode reduzir a qualidade de 
vida do estudante, resultando em perda do controle pessoal, dificuldade na capacidade de aprendizagem consequentemente menor produtividade, diminuição do desempenho acadêmico e tentativa de suicídio (Dagnew, 2020). Os estudos de Supartini et al. (2016), Oyekcin (2017) e Knipe et al. (2018) mostram que 5,77\%, 15,1\% e $16 \%$ respectivamente, dos alunos apresentaram pensamento suicida.

Dos estudos observados, alguns ainda abordam que os estudantes de ciências da saúde são sujeitos ao estresse de fontes possivelmente relacionadas ao seu treinamento educacional, resultando em problemas significativos de saúde mental (Delara, 2015) e que se encontram em maior risco de depressão (Nahas et al., 2019). Esses estudantes expõem-se a eventos estressores frequentemente durante o seu curso e, por conta disso, a sua saúde emocional também impacta sobre sua relação com os pacientes (Leão et al., 2018).

Os resultados do estudo de Delara (2015) mostram que 100\% dos estudantes da área de saúde relataram apresentar depressão. O estudo de Arbués et al. (2019) demonstrou que a maioria dos participantes com depressão cursava graduação na área da saúde $(58,3 \%)$. Outros estudos ainda mostram que estudantes de medicina apresentaram alta prevalência de depressão, quando comparados aos demais estudantes universitários (Lei et al., 2016; Džubur et al., 2018; Chan, 2020). Um dos fatores identificados consiste na tensão para passar nas provas, avaliações e trabalhos (Rehmani, 2018). Outros estudos mostram altos níveis de sintomas depressivos em estudantes do curso de enfermagem (Cheung et al., 2016; Mitchell, 2018), os quais também são prevalentes em estudantes do curso de Fisioterapia (Leão et al., 2018).

Com relação a uma comparação com a população geral, os estudos destacam menor prevalência de problemas de saúde mental entre estudantes de ciências da saúde e que isso pode ser explicado, pelo menos em parte, pelo fato de o nível educacional ser um fator protetor contra problemas psicológicos, como depressão e ansiedade (Tayefi et al., 2019). No entanto, alguns autores destacam e reforçam a importância da prevenção, incluindo a detecção e o tratamento precoce de transtornos mentais, a fim de reduzir a prevalência desses indicadores entre os estudantes, sendo necessária a mudança no estilo de vida para equilibrar a vivência acadêmica e o bem-estar pessoal (Cheung et al., 2016). Além disso, deve-se proporcionar ao estudante de saúde uma formação mais humanizada e completa, na qual o estudante seja atendido em suas 
necessidades pedagógicas e emocionais, de forma a estarem técnica e emocionalmente mais bem preparados e mais saudáveis para lidar com a saúde dos seus pacientes (Leão et al., 2018). É importante destacar também que esse atendimento e acolhimento das necessidades pedagógicas e emocionais dos estudantes pode ser garantido mediante o desenvolvimento de políticas públicas ou das instituições privadas para assistir essa população.

\section{Referências}

Arbués, E. R.; Abadía, B. M.; López, J. M. G. et al. (2019). Eating behavior and relationships with stress, anxiety, depression and insomnia in university students. Nutricion. Hospitalaria, v. 36, n. 6, p. 1339-1345. https://doi.org/10.20960/nh.02641.

Badrasawi, M. M. \& Zidan, S. J. (2019). Binge eating symptoms prevalence and relationship with psychosocial factors among female undergraduate students at Palestine Polytechnic University: a cross-sectional study. Journal of Eating Disorders, v. 7, n. 1, p. 1-8. https://doi.org/10.1186/s40337-019-0263-1.

Bell, C. C. (1994). DSM-IV: diagnostic and statistical manual of mental disorders. Jama, v. 272, n. 10, p. 828-829. https://doi.org/10.1001/jama.1994.03520100096046.

Chan, H. W. Q. \& Sun, C. F. R. (2020). Irrational beliefs, depression, anxiety, and stress among university students in Hong Kong. Journal of American Collage Health, v. 1, n. 15, p. 1-15. https://doi.org/10.1080/07448481.2019.1710516.

Cheung, T.; Wong, S.Y.; Wong, K.Y. et al. (2016). Depression, anxiety and symptoms of stress among baccalaureate nursing students in Hong Kong: a cross-sectional study. International Journal of Environmental Research and Public Health, v. 13, n. 8, p. 779. https://doi.org/10.3390/ijerph13080779.

Cuttilan, A. N.; Sayampanathan, A. A.; Ho, R. C. (2016). Mental health issues amongst medical students in Asia: a systematic review [2000-2015]. Annals of Translational Medicine, v. 4, n. 4. https://doi.org/10.3978/j.issn.2305-5839.2016.02.07.

Cybulski, C. A.; Mansani, F. P. (2017). Análise da depressão, dos fatores de risco para sintomas depressivos e do uso de antidepressivos entre acadêmicos do curso de medicina da Universidade Estadual de Ponta Grossa. Revista Brasileira de Educação Médica, Ponta Grossa, v. 4, n. 1, p. 92-101. http://doi.org/10.1590/1981-52712015v41n1rb20160034.

Dagnew, B.; Dagne, H. \& Andualem, Z. (2020). Depression and its determinant factors among University of Gondar medical and health science students, Northwest Ethiopia: institutionbased cross-sectional study. Neuropsychiatric Disease and Treatment, v. 16, p. 839-845. 
http://doi.org/10.2147/NDT.S248409.

Delara, M. \& Woodgate, R. L. (2015). Psychological distress and its correlates among university students: a cross-sectional study. Journal of Pediatric and Adolescent Gynecology, v. 28, n. 4, p. 240-244. https://doi.org/10.1016/j.jpag.2014.08.012.

Desouky, D. E.; Zaid, H. A.; Taha, A. A. (2019). Migraine, tension-type headache, and depression among Saudi female students in Taif University. Journal of the Egypt Public Health Association, v. 94, n. 1, p. 1-9. https://doi.org/10.1186/s42506-019-0008-7.

Dinis, J. \& Bragança, M. (2018). Quality of sleep and depression in college students: a systematic review. Sleep Science, v. 11, n. 4, p. 290-301. https://doi.org/10.5935/1984$\underline{0063.20180045 .}$.

Džubur, A.; Abdulahović, D.; Kurspahić-Mujčić, A. et al. (2018). Depressive symptoms among Sarajevo University students: prevalence and socio-demographic correlations. Acta Medica Academica, v. 47, n. 2, p. 155-164. http://doi.org/10.5644/ama2006-124.227.

Erić, L; Radovanović, Z; Jevremović, I. (1988). Mental disorders among Yogoslav medical students. British Journal of Psychiatry, v. 152, v. 1, p. 127-129. https://doi.org/10.1192/bjp.152.1.127.

Fernandes, M. A.; Vieira, F. E. R.; Silva, J. S. et al. (2018). Prevalence of anxious and depressive symptoms in college students of a public institution. Revista Brasileira de Enfermagem, v. 71, n. 5, p. 2169-2175. http://doi.org/10.1590/0034-7167-2017-0752.

Fiorotti, K. P.; Rossoni, R. R.; Borges, L. H. et al. (2010). Transtornos mentais comuns entre os estudantes do curso de medicina: prevalência e fatores associados. Jornal Brasileiro Psiquiatria. Vitória, v. 59, n. 1, p. 17-23. https://doi.org/10.1590/S0047-20852010000100003.

Flesch, B. D.; Houvèssou, G. M.; Munhoz, T. N. et al. (2020). Major depressive episode among university students in Southern Brazil. Revista de Saúde Pública, v. 54, p. 11. https://doi.org/10.11606/s1518-8787.2020054001540.

Giglio, J. S. Bem-estar emocional em estudantes universitários: um estudo preliminar. Tesedoutorado. Departamento de psicologia Médica e Psiquiatria. UNICAMP, São Paulo, Brasil. 171p. $1976 . \quad$ Disponível em http://repositorio.unicamp.br/bitstream/REPOSIP/313650/1/Giglio_JoelSales_D.pdf. Acessado em 15 de abril de 2020.

Gonçalves, A. M. C.; Teixeira, M. T. B.; Gama, J. R. A. et al. (2017). Prevalência de depressão e fatores associados em mulheres atendidas pela Estratégia de Saúde da Família. Jornal Brasileiro de Psiquiatria, v. 67, n. 2, p. 101-109. https://doi.org/10.1590/0047-2085000000192.

Grasdalsmoen, M.; Eriksen, H. R.; Lønning, K. J. et al. (2020). Physical exercise, mental health problems, and suicide attempts in university students. BMC Psychiatry, v. 20, p. 1-11. 
https://doi.org/10.1186/s12888-020-02583-3.

Gul, F.; Yuefen, W.; Ullah, I. et al. (2020). Study of depression in university students in Pakistan. Journal of the Pakistan Medical Association, v. 70, n. 4, p. 650-654. https://doi.org/10.5455/JPMA.15757.

Hambisa, M. T; Derese, A; Abdeta, T. (2020). Depressive symptoms among Haramaya University students in Ethiopia: a cross-sectional study. Depression Research and Treatment, v. 2020. https://doi.org/10.1155/2020/5027918.

Hakami, R. M. (2018). Prevalence of psychological distress among undergraduate students at Jazan University: a cross-sectional study. Saudi Journal of Medicine and Medical Science, v. 6, n. 2, p. 82-88. https://doi.org/10.4103/sjmms.sjmms_73_17.

Kilinç, G.; Rukuye, A.; Gülsen, G. et al. (2020). The relationship between depression and loneliness levels of the students at the faculty of health sciences and the factors affecting them. Perspectives in Psychiatric Care, v. 56, n. 2, p. 431-438. https://doi.org/10.1111/ppc.12452.

Knipe, D.; Maughan, C.; Gilbert, J. et al. (2018). Mental health in medical, dentistry and veterinary students: cross-sectional online survey. BJPsych Open, v. 4, n. 6, p. 441-446. https://doi.org/10.1192/bjo.2018.61.

Leão, A. M.; Gomes, I. P.; Ferreira, M. J. M. et al. (2018). Prevalence and factors associated with depression and anxiety among university students in the field of health in a large urban center in the Northeast of Brazil. Revista Brasileira de Educação Médica, v. 42, n. 4, p. 55-65. https://doi.org/10.1590/1981-52712015v42n4rb20180092.

Lee, E. \& Kim, Y. (2019). Effect of university students' sedentary behavior on stress, anxiety, and depression. Perspectives in Psychiatric Care, v. 55, n. 2, p.164-169. https://doi.org/10.1111/ppc.12296.

Lei, X. Y.; Xiao, L. M.; Liu, Y. N. et al. (2016). Prevalence of depression among Chinese university students: a meta-analysis. Plos One, v. 11, n. 4, p. e0153454. https://doi.org/10.1371/journal.pone.0153454.

Li, W.; Meng, X.; Xu, Z. et al. (2015). Prevalence, correlates of major depression: a mental health survey among undergraduates at a mainland Chinese university. Asia Pacific Psychiatry, v. 8, n. 3, p. 206-214. https://doi.org/10.1111/appy.12202.

López, R. B; Navarro, N. M; Astorga, A. C. (2017). Relationship between personality organization and the prevalence of symptoms of depression, anxiety and stress among university students in health careers in the region of Coquimbo, Chile. Revista Colombiana de Psiquiatria, v. 46, n. 4, p. 203-208. http://doi.org/10.1016/j.rcp.2016.07.005.

Lun, K.W.; Chan, C. K.; Ip, P. K. Y. et al. (2018). Depression and anxiety among university students in Hong Kong. Hong Kong Medical Journal, v. 24, n. 5, p. 466-472. 
https://doi.org/10.12809/hkmj176915.

Mboya, I. B.; John, B.; Kibopile, E.S. et al. (2020). Factors associated with mental distress among undergraduate students in northern Tanzania. BMC Psychiatry, v. 20, n. 1, p. 28. https://doi.org/10.1186/s12888-020-2448-1.

Ménard, C.; Hodes, G. E.; Russo, S. J. (2016). Pathogenesis of depression: Insights from human and rodent studies. Neuroscience, v. 3, n. 321, p. 138-162. https://doi.org/10.1016/j.neuroscience.2015.05.053.

Milic, M.; Gazibara, T.; Pekmezovic, T. et al. (2020). Tobacco smoking and health-related quality of life among university students: mediating effect of depression. PLoS One, v. 15, n. 1, p. e0227042. https://doi.org/10.1371/journal.pone.0227042.

Mitchell, A. E. P. (2018). Psychological distress in student nurses undertaking an educational programme with professional registration as a nurse: their perceived barriers and facilitators in seeking psychological support. Journal of Psychiatric and Mental Health Nursing, v. 18, n. 4, p. 258-269. https://doi.org/10.1111/jpm.12459.

Nahas, A. R. M. F.; Elkalmi, M. E.; Al-Shami, A. M. et al. (2019). Prevalence of depression among health sciences students: findings from a public university in Malaysia. Journal of Pharmacy and Bioallied Sciences, v. 11, n. 2, p. 170-175. https://doi.org/10.4103/jpbs.JPBS_263_18.

Ngin, C.; Pal1, K.; Tuot, S. et al. (2018). Social and behavioural factors associated with depressive symptoms among university students in Cambodia: a cross-sectional study. BMJ Open, v. 8, n. 9, p. e019918. http://doi.org/10.1136/bmjopen-2017-019918.

OPAS/OMS - Organização Pan-Americana de Saúde. (2018). Folha informativa - Depressão. Disponível em: https://www.paho.org/bra/index.php?option=com_content $\& v i e w=$ article\&id=5635:folhainformativa-depressao\&Itemid=1095. Acesso em: 24 de maio de 2019.

Othman, N.; Ahmad, F.; El Morr, C. et al. (2019). Perceived impact of contextual determinants on depression, anxiety and stress: a survey with university students. International Journal of Mental Health Systems, v. 13, n. 1, p. 1-9. https://doi.org/10.1186/s13033-019-0275-x.

Oyekcin, D. G; Sahin, E. M; Aldemir, E. (2017). Mental health, suicidality and hopelessness among university students in Turkey. Asian Journal of Psychiatry, v. 29, p. 185-189. https://doi.org/10.1016/j.ajp.2017.06.007.

Oztasan, N; Ozyrek, P; Kilic, I. (2016). Factors associated with health-related quality of life among university students in Turkey. Mater Sociomed, v. 28, n. 3, p. 210-214. https://doi.org/10.5455/msm.2016.28.210-214.

Piumatti, G. (2018). Motivation, health-related lifestyles and depression among university 
students: a longitudinal analysis. Psychiatry Research, v. 260, p. 412-417. https://doi.org/10.1016/j.psychres.2017.12.009.

Rehmani, N; Khan, Q. A; Fatima, S.S. (2018). Stress, anxiety and depression in students of a private medical school in Karachi, Pakistan. Pakistan Journal of Medical Sciences, v. 34, n. 3, p. 696-701. https://doi.org/10.12669/pjms.343.14664.

Rombaldi, A. J.; Silva, M. C.; Gazalle, F. K. et al. (2010). Prevalência e fatores associados a sintomas depressivos em adultos do sul do Brasil: estudo transversal de base populacional. Revista Brasileira de Epidemiologia, v. 13, n. 4, p. 620-629. http://dx.doi.org/10.1590/S1415790X2010000400007.

Rossi, J. L.; Jiménez, J. P.; Barros, P. et al. (2019). Depressive symptomatology and psychological well-being among Chilean university students. Revista Médica de Chile, v. 147, n. 5, p. 579-588. https://doi.org/10.4067/s0034-98872019000500579.

Ruisoto, P.; Cacho, R.; López-Goñi, J. J. et al. (2016). Prevalence and profile of alcohol consumption among university students in Ecuador. Gaceta Sanitaria, v. 30, n. 5, p. 370-374. https://doi.org/10.1016/j.gaceta.2016.02.008.

Santangelo, O. E; Provenzano. S; Firenze, A. (2018). Anxiety, depression and risk consumption of alcohol in a sample of university students. Rivista di Psichiatria, v. 53, n. 2, p. 88-94. https://doi.org/10.1708/2891.29157.

Santos, C. M. C.; Pimenta, C. A. M.; Nobre, M. R. C. (2007). A estratégia pico para a construção da pergunta de pesquisa e busca de evidências. Revista Latino-Americana de Enfermagem, v. 15, n. 3, p. 508-511. 10.1590/S0104-11692007000300023.

Schlarb, A. A.; Claßen, M.; Grünwald, J. et al. (2017). Sleep disturbances and mental strain in university students: results from an online survey in Luxembourg and Germany. International Journal of Mental Health Systems, v.11, n. 1,p.1-10. https://doi.org/10.1186/s13033-017-01319.

Segal, B. E. (1966). Epidemiology of emotional disturbance among college undergraduates: a review and analysis. Journal Nervous and Mental Disease, v. 143, n. 4, p. 348-362. https://doi.org/10.1097/00005053-196610000-00006.

Seo, E. J.; Ahn, J.; Hayman, L. L. et al. (2018). The association between perceived stress and quality of life in university students: the parallel mediating role of depressive symptoms and health-promoting behaviors. Asian Nursing Research, v. 12, n. 3, p. 190-196. https://doi.org/10.1016/j.anr.2018.08.001.

Sibnath, D.; Parveen, R.; Banu, S. T. et al. (2016). Depression among Indian university students and its association with perceived university academic environment, living arrangements and personal issues. Asian Journal Psychiatry, v. 23, p. 108-117. https://doi.org/10.1016/j.ajp.2016.07.010. 
Silva, M. T. et al. (2012). Antidepressivos no transtorno depressivo maior em adultos. Boletim Brasileiro de Avaliação de Tecnologias em Saúde. Ano VI, n. 18.

Supartini, A.; Honda, T.; Basri, N. A. et al. (2016). The impact of sleep timing, sleep duration, and sleep quality on depressive symptoms and suicidal ideation amongst Japanese freshmen: The EQUSITE study. Sleep Disorders, v. 2016. https://doi.org/10.1155/2016/8737654.

Tayefi, B.; Eftekhar, M., Tayefi, M. et al. (2020). Prevalence and socio-demographic correlates of mental health problems among iranian health sciences students. Academic Psychiatry, v. 44, n. 1, p. 73-77. https://doi.org/10.1007/s40596-019-01121-y.

Tran, A.; Tran, L.; Geghre, N. et al. (2017). Health assessment of French university students and risk factors associated with mental health disorders. Plos One, v. 12, n. 11. p. e0188187. https://doi.org/10.1371/journal.pone.0188187.

Tuyen, N. T. H; Dat, T. Q; Nhung, H. T. H. (2019). Prevalence of depressive symptoms and its related factors among students at Tra Vinh University, Vietnam in 2018. AIMS Public Health, v. 6, n. 3, p. 307-319. https://doi.org/10.3934/publichealth.2019.3.307.

Uher, R.; Payne, J. L.; Pavlova, B. et al. (2014). Major depressive disorder in DSM-5: implications for clinical practice and research of changes from DSM-IV. Depress Anxiety, v. 31, n. 6, p. 459-471. https://doi.org/10.1002/da.22217.

\section{Direitos autorais (Copyrights)}

Financiamento: Esta pesquisa não recebeu nenhum financiamento.

Conflitos de interesse: Os autores declaram não haver conflitos de interesse.

Aprovação do comitê de ética: Não aplicável.

Disponibilidade dos dados da pesquisa: Todos os dados gerados ou analisados neste estudo estão incluídos no manuscrito ou na seção 'materiais complementares/quando houver).

Contribuição dos autores: Idealização, investigação e execução da pesquisa: SILVA, T. V. S.; OLIVEIRA, L. M. Condução, revisão metodológica, correção e revisão total do manuscrito: SILVA, T. V. S.; CORREIA, L. S.; OLIVEIRA, L. M. 